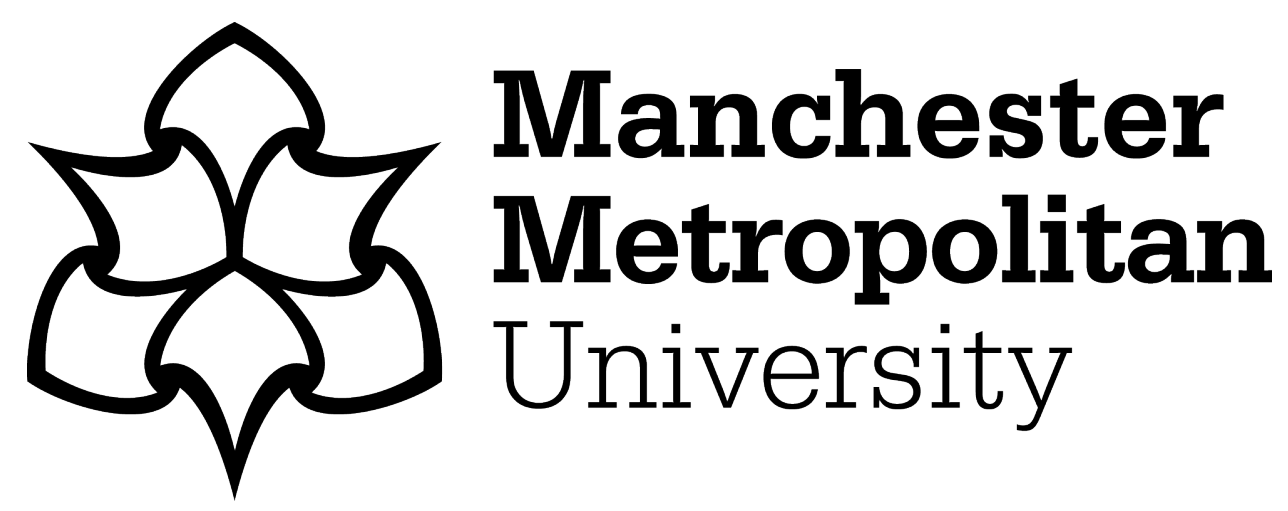

Lin, Zaibin, Chen, Hao, Qian, Ling ORCID logoORCID: https://orcid.org/0000-0002-9716-2342, Ma, Zhihua, Causon, Derek and Mingham, Clive (2021) Simulating focused wave impacts on point absorber wave energy converters. Proceedings of the Institution of Civil Engineers

- Engineering and Computational Mechanics, 174 (1). pp. 19-31. ISSN 1755-0777

Downloaded from: https://e-space.mmu.ac.uk/627190/

Version: Accepted Version

Publisher: Thomas Telford Ltd.

DOI: https://doi.org/10.1680/jencm.19.00038

Please cite the published version 


\section{Simulating focused wave impacts on point absorber wave energy converters}

\author{
Zaibin Lin PhD \\ Research Associate, Centre for Mathematical Modelling and Flow \\ Analysis, Department of Computing and Mathematics, Manchester \\ Metropolitan University, Manchester, M1 5GD, United Kingdom \\ Hao Chen PhD \\ Assistant Professor, School of Engineering, University of Glasgow, \\ Glasgow G12 8QQ, United Kingdom \\ Ling Qian PhD \\ Professor, Centre for Mathematical Modelling and Flow Analysis, \\ Department of Computing and Mathematics, Manchester Metropolitan \\ University, Manchester, M1 5GD, United Kingdom (corresponding \\ author: I.qian@mmu.ac.uk)
}

\author{
Zhihua Ma PhD \\ Senior Lecturer, Centre for Mathematical Modelling and Flow \\ Analysis,Department of Computing and Mathematics, Manchester \\ Metropolitan University, Manchester, M1 5GD, United Kingdom \\ Derek Causon PhD \\ Professor, Centre for Mathematical Modelling and Flow Analysis, \\ Department of Computing and Mathematics, Manchester Metropolitan \\ University, Manchester, M1 5GD, United Kingdom \\ Clive Mingham \\ Professor, Centre for Mathematical Modelling and Flow Analysis, \\ Department of Computing and Mathematics, Manchester Metropolitan \\ University, Manchester, M1 5GD, United Kingdom
}

This work contributes to the CCP-WSI Blind Test Series 2 by numerically investigating the dynamic response of two simplified point absorber wave energy converters (a hemispherical-bottom cylinder and a cylinder with moon-pool) under the action of focused waves of varying steepness. The open source toolbox OpenFOAM along with its new overset grid functionality is applied and evaluated for the complex flow problem involving both large free surface deformations and large amplitude motions of floating objects. The quality of the numerically generated focused wave groups is first examined and validated against the experimental data. The effects of both wave steepness and the moon-pool on the dynamic responses and mooring loads of the simplified wave energy converters (WECs) are then analyzed.

\section{Introduction}

During the last few decades, to harness the energy of ocean waves, a number of prototypes of Wave Energy Converters (WECs) have been proposed and evaluated both experimentally and numerically. As one of the typical designs of WECs, point absorbers work best for the scenarios where wave amplitude is large and its frequency is close to the natural frequency of the device. Under these conditions especially with the occurrence of wave breaking the interactions between waves and point absorber WECs becomes highly nonlinear leading to large motion responses of the WECs, which cannot be adequately captured by potential flow based models. Therefore, to model such flow problems, Computational Fluid Dynamics (CFD) models based on the solutions of the full Navier-Stokes equations are required.

To investigate the capabilities of different CFD models for predicting the interaction between regular waves and WECs, Westphalen et al. (2010) compared the numerical results from both Eulerian and Lagrangian CFD models. It was concluded that overall good agreements have been obtained by all CFD models despite high computational cost. Recently, Devolder et al. (2016) developed a Numerical Wave Tank (NWT) solver that is able to accurately predict the interaction of wave trains and a WEC with vertical motion only. Afterwards, Devolder et al. (2018) applied the NWT to investigate the hydrodynamics of multiple floating point absorber WECs under regular waves. The capability of the NWT for accurately capturing radiated wave fields, forces, heave motion has been demonstrated through comparison with the experimental measurements. Apart from these traditional NS-VoF based NWT solvers, Domínguez et al. (2019) coupled a smooth particle hydrodynamics (SPH) based model (DualSPHysics) with a dynamic mooring line model (MoorDyn) to investigate the motion response of a floating structure in regular waves and good agreements between numerical and experimental data have been achieved.

Furthermore, the survivability of WECs under extreme wave conditions needs to be systematically investigated (Ransley et al., 2017). To this end, a large body of work on numerically modelling of hydrodynamic response of floating WECs under extreme waves can be found in the literature (Yu and Li, 2013; Palm et al., 2016; Hu et al., 2011), in which the focused wave groups formed 
by the superposition of a large number of wave components of varying frequencies and amplitudes have been used to represent extreme waves. At the same time laboratory model tests have also been conducted in order to understand dynamic response of WECs as well as mooring loads in focused waves (Hann et al., 2015). It is found that for the case of a hemispherical-bottom WEC, an increase in focusing wave crests leads to larger surge and pitch motion responses. On the other hand, moon-pools have been commonly used in offshore and marine operations, e.g. in launching or recovery of subsea devices from ships and offshore platforms. Inside the moon-pool piston-like wave resonance often occurs, resulting in significant amplification of free surface elevation.

This work contributes to the CCP-WSI Blind Test Series 2, which is designed to provide an understanding of the required model fidelity for modelling the behaviour of point absorber WECs under focused wave groups. Specifically, the new overset mesh solver in OpenFOAM, given its wide use in both academic research and industrial applications, is evaluated for modelling such flow problems, which provides a reasonably high-fidelity contribution to the comparative study. For simulating dynamic response of a floating structure in waves, overset mesh method provides an effective alternative to dynamic mesh approach. The previous studies in Ma et al. (2018) and Chen et al. (2019b) demonstrated that for dynamic mesh method when large body displacement occurs, such as in water entry problems, mesh quality and hence solution accuracy may deteriorate due to unacceptably large mesh distortion, while the issue can be conveniently resolved by using overset mesh method. Two geometries of the simplified WEC are considered, i.e. a hemispherical-bottom cylinder and a hollow cylinder with moon-pool. In the following sections, the mathematical formulae of numerical models are presented, followed by a discussion of the numerical numerical results and a brief summary of the work.

\section{Mathematical formulae}

\subsection{Governing equations}

In this numerical study, the fluid dynamics is governed by incompressible Navier-Stokes equations which are spatially discretised by the Finite Volume Method as implemented in the open-source toolbox OpenFOAM. A two-phase model, together with the Volume of Fluid method, is applied to describe both the water and air flow as well as their interface. The governing equations for this model are given below:

(1) $\nabla \cdot \boldsymbol{u}=0$

$$
\frac{\partial \rho \boldsymbol{u}}{\partial t}+\nabla \cdot(\rho \boldsymbol{u}) \boldsymbol{u}-\nabla \cdot \mu \nabla \boldsymbol{u}=-\nabla p^{*}-(\boldsymbol{g} \cdot \boldsymbol{x}) \nabla \rho
$$

$$
\frac{\partial \alpha}{\partial t}+\nabla \cdot \boldsymbol{u} \alpha+\nabla \cdot\left(\boldsymbol{u}_{r} \alpha(1-\alpha)\right)=0
$$

where $\boldsymbol{u}$ is the velocity field, $\rho$ is fluid density, $t$ is time, $\mu$ is dynamic viscosity of fluid, $p^{*}=p-\rho \boldsymbol{g} \cdot \boldsymbol{x}$ is the pressure in excess of static pressure, $\boldsymbol{g}$ is gravitational acceleration, $\boldsymbol{x}=$ $(x, y, z)$ is the Cartesian coordinate system, $\alpha$ is the volume fraction, and $\boldsymbol{u}_{\boldsymbol{r}}$ is compressive velocity field (Berberović et al., 2009), which maintains the sharp water-air interface.

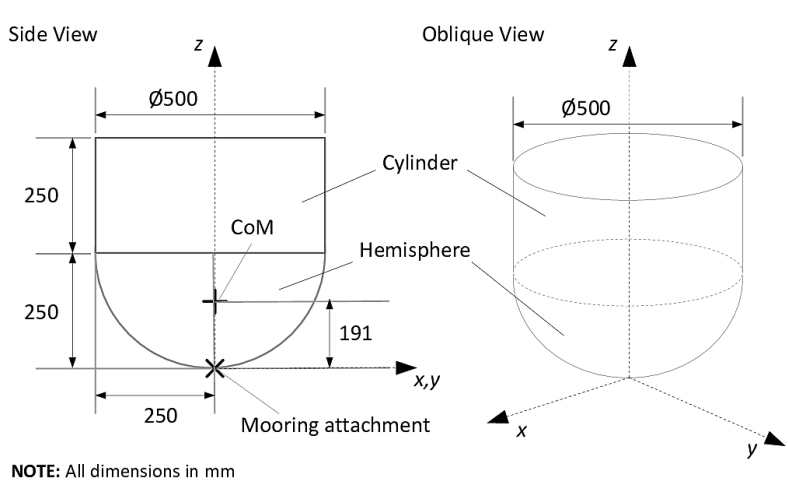

Figure 1. Sketch of the geometry and dimensions of a hemispherical-bottom cylinder. CoM: Centre of Mass. Source: CCP-WSI Blind Test Series 2 (2020)

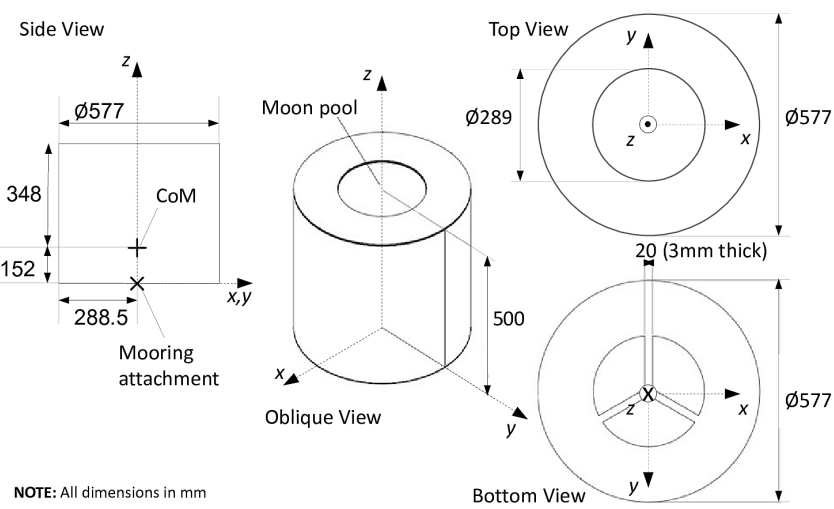

Figure 2. Sketch of the geometry and dimensions of a cylinder with moon-pool. CoM: Centre of Mass. Source: CCP-WSI Blind Test Series 2 (2020)

\subsection{Overset mesh}

As a recently released functionality in OpenFOAM, overset mesh solver is adopted in this study since it is capable of accurately capturing large amplitude body motions, such as in water entry problems, which may not be adequately resolved by dynamic (moving) mesh approach, also implemented in OpenFOAM, due to potentially large distortion and hence reduced quality of the mesh. Within the overset mesh approach, a composite mesh system is introduced with multiple layers of meshes: one is the background 
mesh and others are the body-fitted meshes - one for each component of the structure(s). During the simulation, the bodyfitted mesh moves with the floating structure as an entity without any mesh deformations, while the background mesh is fixed. These two mesh layers exchange data by interpolation at every time step. Readers are referred to Chen et al. (2019b) and Ma et al. (2018) for more details on overset mesh implementations.

\subsection{Rigid body motion solver}

The six degrees of freedom of WECs are numerically solved by a rigid body motion solver in OpenFOAM (sixDoFRigidBodySolver), whereas a linear spring is used in the mooring system to restrain WECs motions in a numerical wave basin. In this solver, two equations, namely linear and angular momentum conservation equations, are numerically solved to provide the acceleration of WECs. An acceleration relaxation factor of 0.9 is selected in order to stabilize the solution during the simulation. The displacement and velocity of WECs are predicted by integrating the acceleration using the Newmark scheme.

\subsection{Numerical wave generation}

Here NewWave type focused wave groups (Tromans et al., 1991; Baldock et al., 1996) are adopted to represent the extreme wave conditions as specified in the Blind Test Series 2. The wave generation boundary conditions are implemented in IHFOAM (Higuera et al., 2013a,b) where the velocity profile and wave amplitude are specified at the inlet boundary on the basis of the second order irregular wave theory (Hu et al., 2016). OpenFOAM v1906 from ESI-OpenCFD is used in the present study.

\section{Results and discussions}

\subsection{Experiments}

The physical experiments for the Blind Tests were conducted in the COAST Laboratory Ocean Basin, University of Plymouth, UK. The wave basin is $35 \mathrm{~m}$ long, $15.5 \mathrm{~m}$ wide, and $3 \mathrm{~m}$ deep. In the experiments, two simplified WECs: a hemispherical-bottomed cylinder as illustrated in Fig. 1 and a cylinder with a moonpool as illustrated in Fig. 2, are tested under the focused wave environments. The corresponding mass properties of the WECs can be found in Table 1. The WECs were moored using a linear spring, which is fixed to the $w$ ave $b$ asin $b$ ottom. The s pring $s$ tiffness of mooring line is $67 \mathrm{~N} / \mathrm{m}$ and the pre-tensions in mooring are $32.07 \mathrm{~N}$ and $31.55 \mathrm{~N}$ for the two cases, respectively. For more details of the experimental setups for the Blind Test, readers are referred to the upcoming paper of Ransley et al. (In review) and CCP-WSI website (CCP-WSI Blind Test Series 2, 2020).

In Table 2, the parameters of focused waves generated in the experiment are listed. The crest heights of all three focused wave groups are kept the same, while the peak frequencies are different. The target waves were formed by linearly superposing 244 wave fronts, whose frequencies are uniformly distributed between $0.10 \mathrm{~Hz}$ and $2 \mathrm{~Hz}$. In the numerical setup, 13 wave gauges

\begin{tabular}{cccccc}
\hline ID & $\begin{array}{c}M \\
{[\mathrm{~kg}]}\end{array}$ & $\begin{array}{c}I_{x x} \\
{\left[\mathrm{kgm}^{2}\right]}\end{array}$ & $\begin{array}{c}I_{y y} \\
{\left[\mathrm{kgm}^{2}\right]}\end{array}$ & $\begin{array}{c}I_{z z} \\
{\left[\mathrm{kgm}^{2}\right]}\end{array}$ & $\begin{array}{c}d \\
{[\mathrm{~m}]}\end{array}$ \\
\hline 1 & 43.674 & 1.62 & 1.62 & 1.143 & 0.322 \\
2 & 61.459 & 3.56 & 3.56 & 3.298 & 0.330 \\
\hline
\end{tabular}

$M$ is the mass of WECs, $I_{x x}, I_{y y}$, and $I_{z z}$ are the moment of inertia. $\mathrm{d}$ is the draft. ID 1 is the hemispherical-bottom cylinder and ID 2 is the cylindrical moon-pool.

Table 1. Mass properties of two wave energy converters

\begin{tabular}{cccccc}
\hline Case & $\begin{array}{c}A \\
{[\mathrm{~m}]}\end{array}$ & $\begin{array}{c}T_{p} \\
{[\mathrm{~s}]}\end{array}$ & $\begin{array}{c}H_{s} \\
{[\mathrm{~m}]}\end{array}$ & $\begin{array}{c}h \\
{[\mathrm{~m}]}\end{array}$ & $\begin{array}{c}k_{p} A \\
{[-]}\end{array}$ \\
\hline 1 & 0.25 & 2.7949 & 0.274 & 3 & 0.128778 \\
2 & 0.25 & 2.5 & 0.274 & 3 & 0.160972 \\
3 & 0.25 & 2.2821 & 0.274 & 3 & 0.193167 \\
\hline
\end{tabular}

Note: $A$ is focal crest height, $T_{p}$ is the wave period for the wave component at peak frequency. $H_{s}$ is significant wave height, $h$ is water depth, and $k_{p} A$ is wave number for the wave component at the peak frequency. Pierson-Moskowitz spectrum is adopted in this study.

Table 2. Test conditions of the focused wave groups

are located based on the laboratory wave gauges layout in Fig. 3, where the buoy was initially located at the focal position of wave group at wave gauge 5 . In the final computations, this wave gauge was removed due to the presence of WECs.

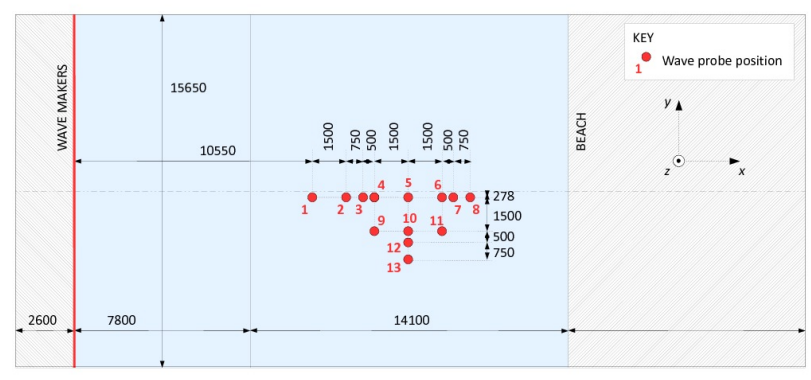

Figure 3. The layout of wave gauges in the COAST Laboratory Ocean Basin, University of Plymouth, the UK. Source: CCP-WSI Blind Test Series 2 (2020)

\subsection{Computational domain and mesh}

The size of the numerical domain is $20 \mathrm{~m}$ long and $6 \mathrm{~m}$ wide, corresponding to $1.9 \lambda_{p}$ ( $\lambda_{p}$ is wave length at peak frequency) and $12 D_{1}$ and $10 D_{2}$ respectively ( $D_{1}$ is the diameter of the hemispherical-bottom WEC and $D_{2}$ is the diameter of the moonpool WEC). The initial water depth of the numerical domain is $3 \mathrm{~m}$, which is the same as that in laboratory. Above the water phase, the height of the air phase is initially set at $1 \mathrm{~m}$ to allow enough 


\begin{tabular}{cccc}
\hline Mesh ID & $\begin{array}{c}\text { Domain size } \\
x \times y \times z\end{array}$ & $\begin{array}{c}\text { Mesh layout } \\
x \times y \times z\end{array}$ & simpleGrading \\
& $20 \mathrm{~m} \times 1 \mathrm{~m} \times 4 \mathrm{~m}$ & $400 \times 1 \times 140$ & $x:(0.55,0.45,0.2)$ \\
& & & $(0.15,0.40,1.0)$ \\
& & & $(0.30,0.15,9.0)$ \\
& & & $z:(0.56,0.15,0.1)$ \\
& & & $(0.32,0.75,1.0)$ \\
M1 & $20 \mathrm{~m} \times 1 \mathrm{~m} \times 4 \mathrm{~m}$ & $530 \times 1 \times 140$ & Same as M1 \\
M3 & $20 \mathrm{~m} \times 1 \mathrm{~m} \times 4 \mathrm{~m}$ & $660 \times 1 \times 140$ & Same as M1 \\
M4 & $20 \mathrm{~m} \times 1 \mathrm{~m} \times 4 \mathrm{~m}$ & $790 \times 1 \times 140$ & Same as M1 \\
\hline
\end{tabular}

Note: 'simpleGrading' as defined in the mesh property in the blockMeshDict of OpenFOAM specifies the mesh expansion ratio.

Table 3. Mesh layouts for mesh sensitivity study

\begin{tabular}{cccc}
\hline $\begin{array}{c}\text { Mesh } \\
\text { ID }\end{array}$ & $\begin{array}{c}\text { Background } \\
x \times y \times z\end{array}$ & simpleGrading & $\begin{array}{c}\text { Overset } \\
x \times y \times z\end{array}$ \\
\hline OSM1 & $530 \times 96 \times 140$ & $y:(0.38,0.1,0.04)$ & $64 \times 64 \times 56$ \\
& & $(0.24,0.8,1)$ & \\
& & $(0.38,0.1,25)$ & \\
OSM2 & $595 \times 108 \times 140$ & Same as OSM1 & $72 \times 72 \times 63$ \\
OSM3 & $660 \times 120 \times 140$ & Same as OSM1 & $80 \times 80 \times 70$ \\
\hline
\end{tabular}

Note: The domain size is $20 \mathrm{~m} \times 5 \mathrm{~m} \times 4 \mathrm{~m}$ in $x, y$, and $z$ direction, respectively. In the $x$ and $z$ directions, the simpleGrading of the background mesh is the same as those in Table 3, and the simpleGrading of the overset mesh is set to 1 .

Table 4. Mesh layouts for mesh sensitivity study of overset mesh solver

space for the potential WECs motion. The numerical wave groups are focused at $12 \mathrm{~m}$ away from the wave generation boundary and at $12 \mathrm{~s}$ in time.

Prior to carrying out the investigation of the interaction between focused waves and WECs, mesh convergence studies are performed to determine the optimal mesh density for focused wave propagation in the NWT. Four different mesh layouts are adopted as listed in Table 3, where all cases were performed in 2-D to reduce computational efforts as only unidirectional waves with $0^{\circ}$ incident angle were used in CCP-WSI Blind Test Series 2 (2020). Therefore, the mesh cell in $y$ direction is set to 1 as shown in Table 3 .

In Fig. 4, the results from all the meshes for wave elevation at Wave Gauge 5 are compared with experimental measurements. It can be seen from Fig. 4(a) that overall good agreements are demonstrated for all mesh layouts, while the discrepancies between the numerical and experimental results after the focal time are likely due to wave reflections from the outlet boundary of the NWT. Detailed comparisons between the results from these mesh layouts are presented in Fig. 4(b), where only very minor difference can be observed between mesh layouts M3 and M4. Thus, in order to balance the computational costs and solution accuracy, the mesh layout M3 in Table 3 is adopted for following simulations.
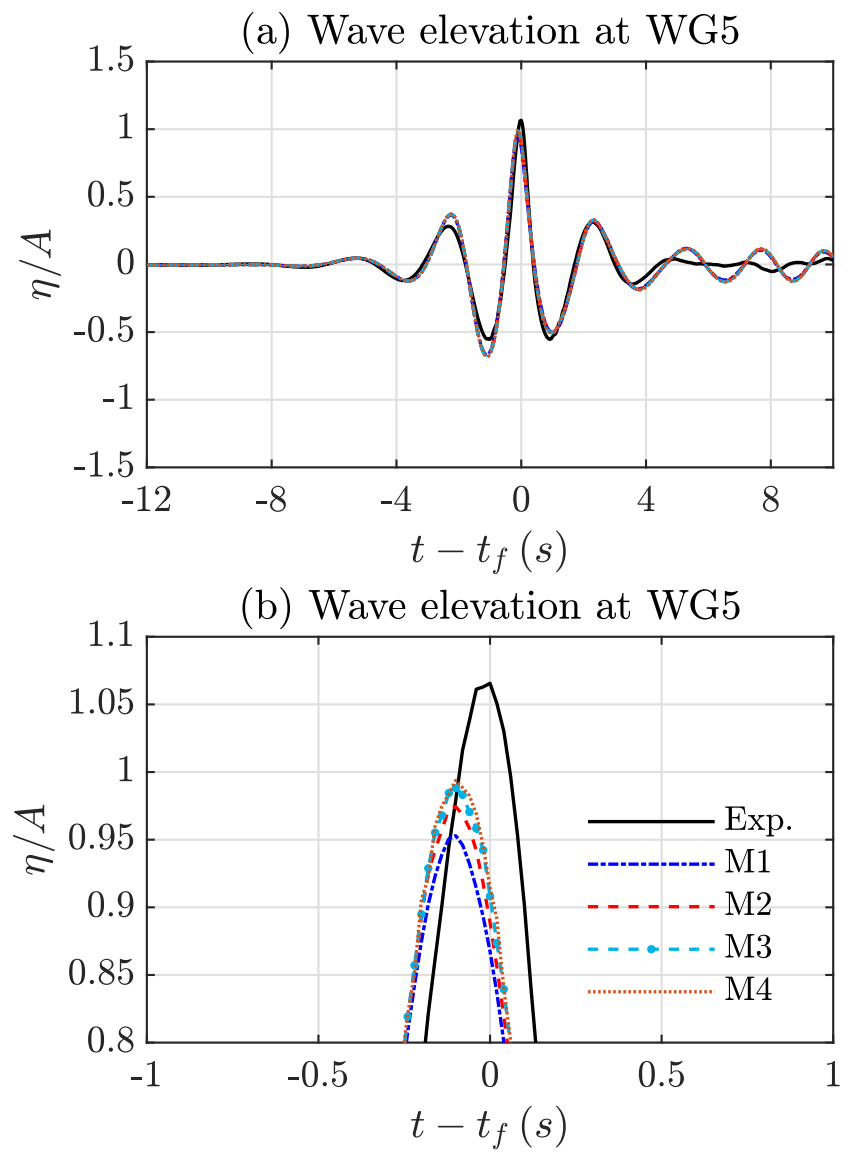

Figure 4. Mesh convergence study of Case 2 in Table 2 with mesh layouts in Table 3.

As an overset grid has been applied in this work, two layers of overlapping meshes, namely the background mesh and the minor body-fitted mesh will be generated. For the background mesh, which covers the entire domain and is fixed during the simulation, it is smoothly refined in the vertical direction $z$, from both the tank bottom and atmospheric boundaries towards the zone with the boundaries of $z=-0.3 \mathrm{~m}$ and $z=0.3 \mathrm{~m}$ with $z=0 \mathrm{~m}$ being the initial interface between water and air. Between the two boundaries, the mesh is evenly spaced. In the two horizontal directions, the mesh is stretched smoothly from the domain sides to the focal location. A utility named snappyHexMesh is used here to produce the minor body-fitted mesh, which cuts the background regular hexahedral cells into tetrahedron cells in order to fit an arbitrary solid boundary. In addition, the meshes are refined in the vicinity of two WECs in all three directions. The mesh layouts near the WECs are shown in Fig. 5(a) and (b) with mesh refinement to better capture motion 
responses, and the overall mesh layout of the NWT is presented in Fig. 5(c).

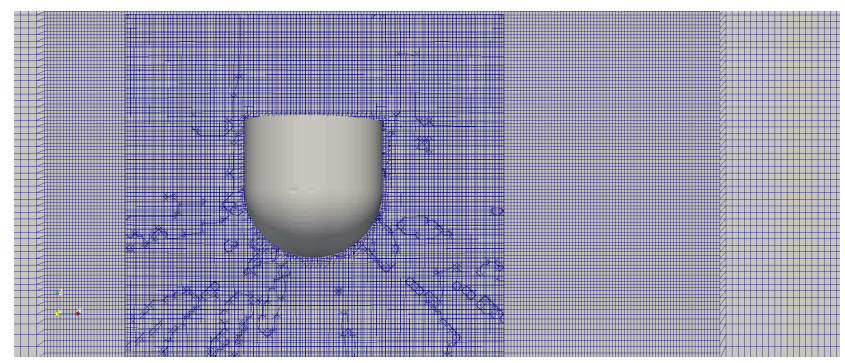

(a) local mesh around the hemispherical-bottom cylinder

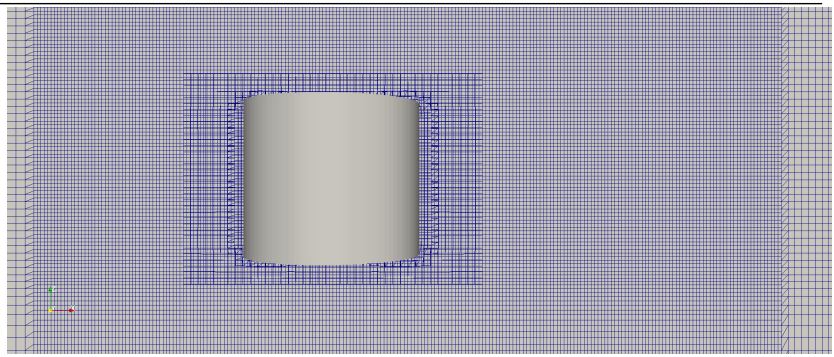

(b) Local mesh around the moon-pool cylinder

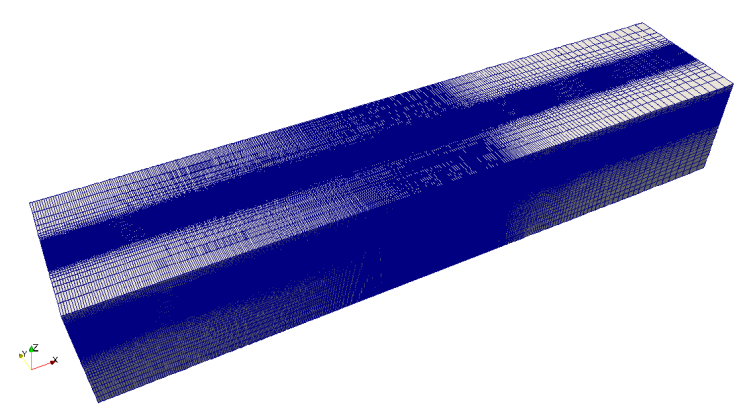

(c) Overall mesh layout of the numerical wave tank

Figure 5. The computational mesh

Based on the aforementioned mesh convergence study for focused wave propagation and the mesh study in Chen et al. (2019c, 2020), three mesh layouts, as listed in Table 4, of the background and overset mesh for a moonpool cylinder are chosen to investigate the mesh convergence of the overset mesh solver. The mesh inside the rectangle zone $(-1-0.8-0.7)(1.50 .80 .7)$ is refined in all three directions in order to provide sufficient mesh resolution to capture the WECs motions in focused waves. The numerical results from the three difference mesh layouts for surge, heave, pitch motion and mooring force are compared in Fig. 6. It can be seen that while the results from the coarse mesh layout (OSM1) show a slight phase difference from those of the medium (OSM2) and fine mesh layouts (OSM3), the difference between the medium (OSM2) and fine mesh layouts (OSM3) is negligible. It can be concluded that good
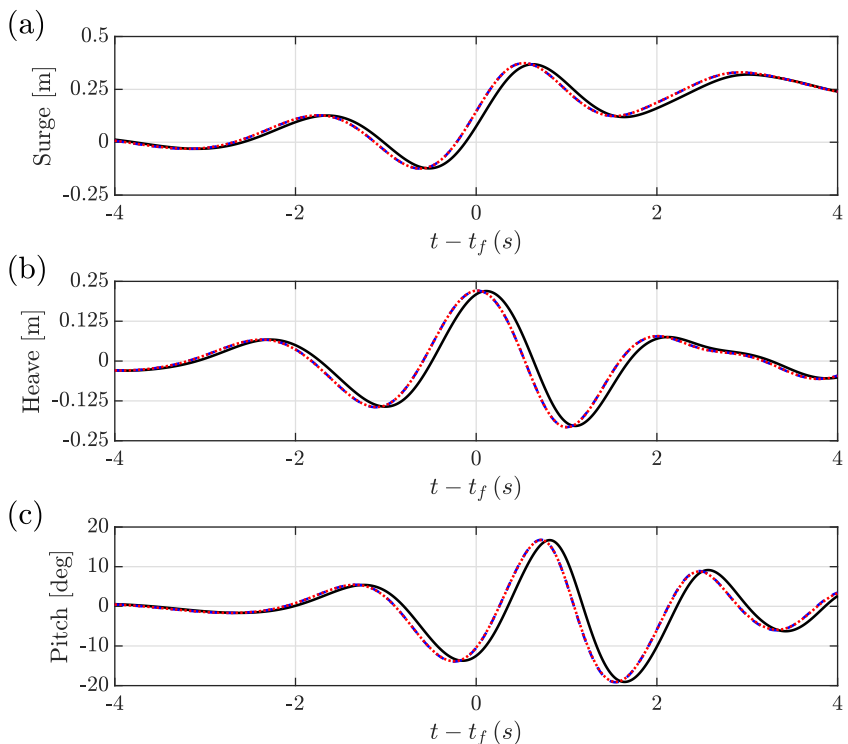

(d)

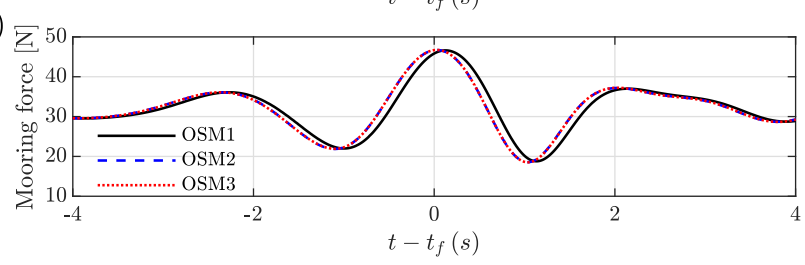

Figure 6. Overset mesh convergence study of Case 2 in Table 2 with mesh layouts in Table 4 for a moonpool cylinder.

mesh convergence has been achieved for the overset mesh solver based on the medium (OSM2) and fine mesh layouts (OSM3). In the following studies in sections 3.5 and 3.6 the fine mesh layout (OSM3) has been adopted for its better performance in the mesh convergence study for focused wave propagation as shown in Fig. 4. More details on mesh setups including mesh cell numbers can be found in Lin et al. (2019) and Table 5.

\subsection{Validation of focused waves generation}

The free surface elevations at Wave Gauges (WGs) 1, 3, 5, and 8 are validated against experimental results in Figs. 7, 8, and 9 for Case 1,2 , and 3, respectively, the relative error defined by $\frac{\eta-\eta_{T}}{A}$ and together with peak amplitude error $\left(E_{p}\right)$ at WG5, where $\eta$ is numerical results and $\eta_{T}$ is target experimental measurements. In general the generated focused wave groups agree well with measured free surface elevations in the COAST wave basin, especially for the Cases 1 and 2, while larger discrepancy wave crest at the focal position can be seen in Fig. 9 for Case 3 with the highest wave steepness. This could be attributed to the fact that the actual focused time and location produced in the laboratory are unknown and different wave generation methods and the domain sizes have been used in the numerical simulations and experimental studies, despite that the theoretical focused time and location for 
wave generation are imposed to wave maker in the laboratory and a linear superposition of wave fronts is adopted in both approaches.
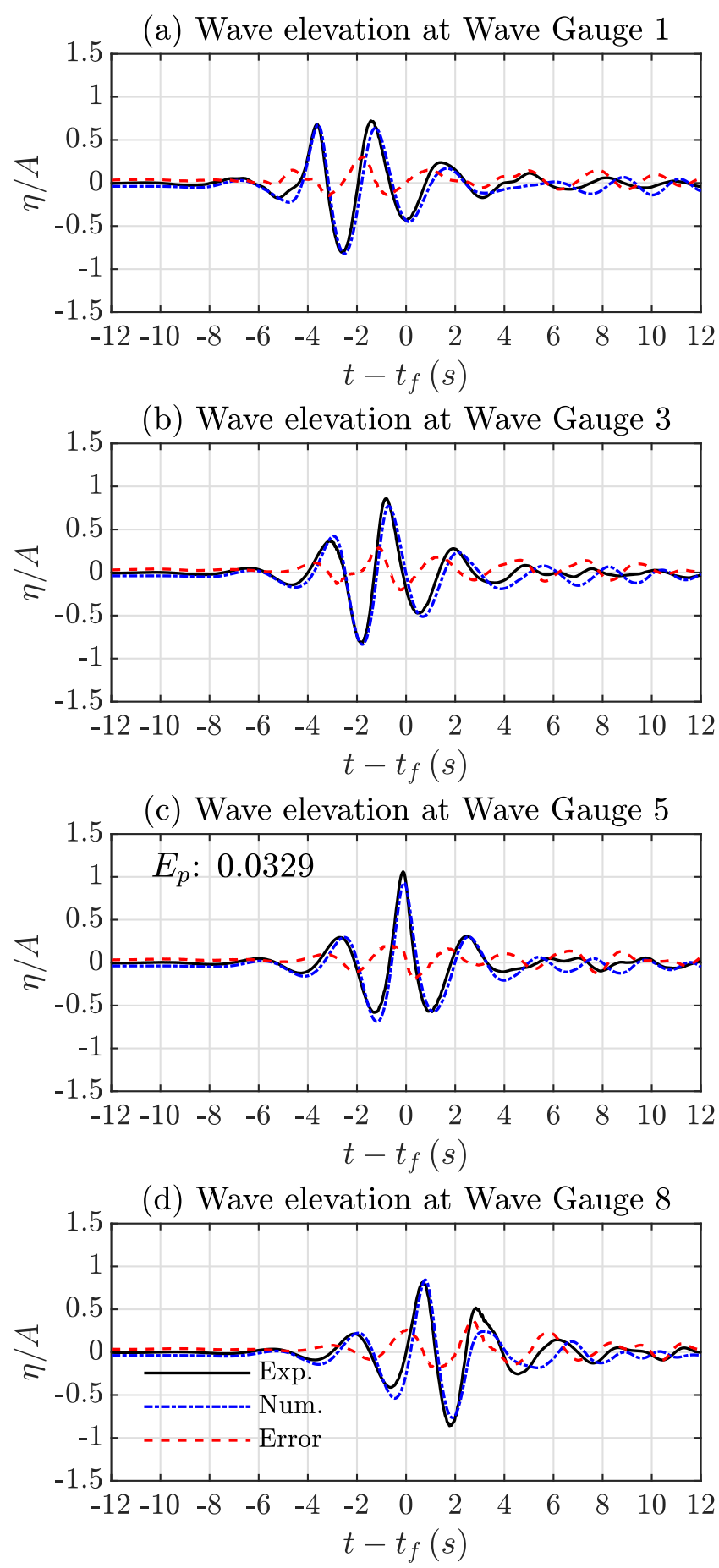

Figure 7. Comparisons between numerical results of Case 1 against experimental data at four Wave Gauges. (a) Wave elevation at Wave Gauge 1

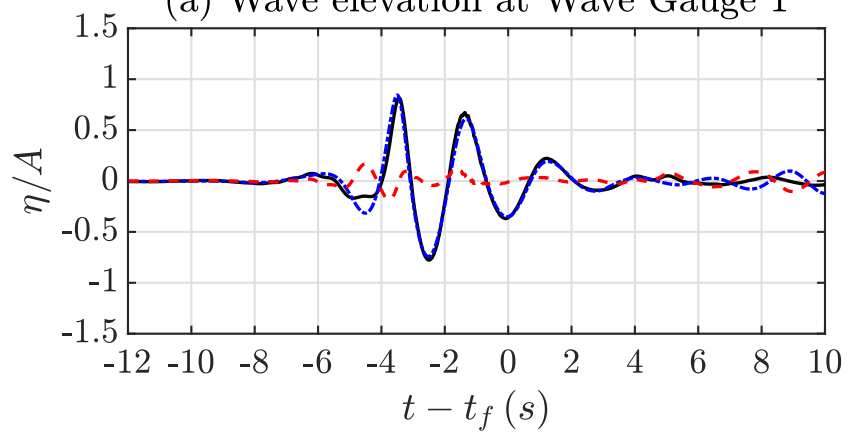

(b) Wave elevation at Wave Gauge 3

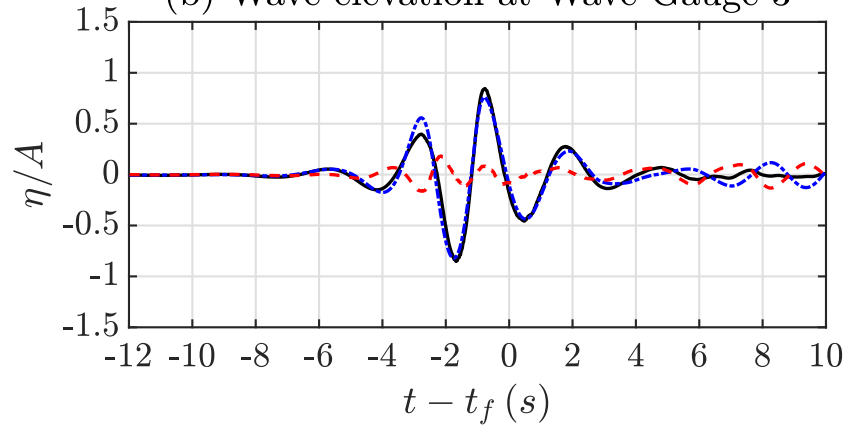

(c) Wave elevation at Wave Gauge 5

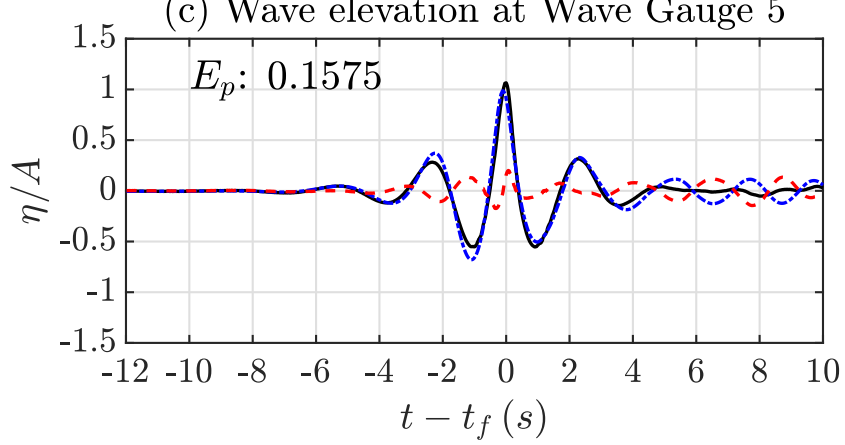

(d) Wave elevation at Wave Gauge 8

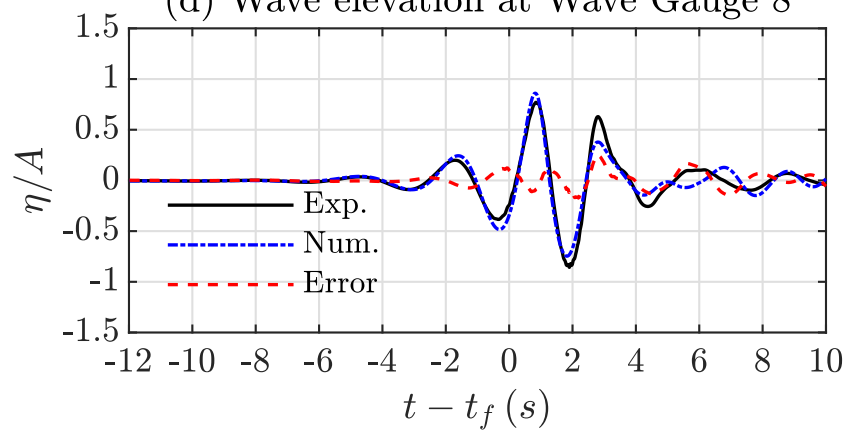

Figure 8. Comparisons between numerical results of Case 2 against experimental data at four Wave Gauges.

\subsection{Effects of wave steepness and geometry} difference on WECs motions

A floating cylinder with moon-pool, subjected to the impact of focused waves with varying peak frequencies, is firstly investigated, 

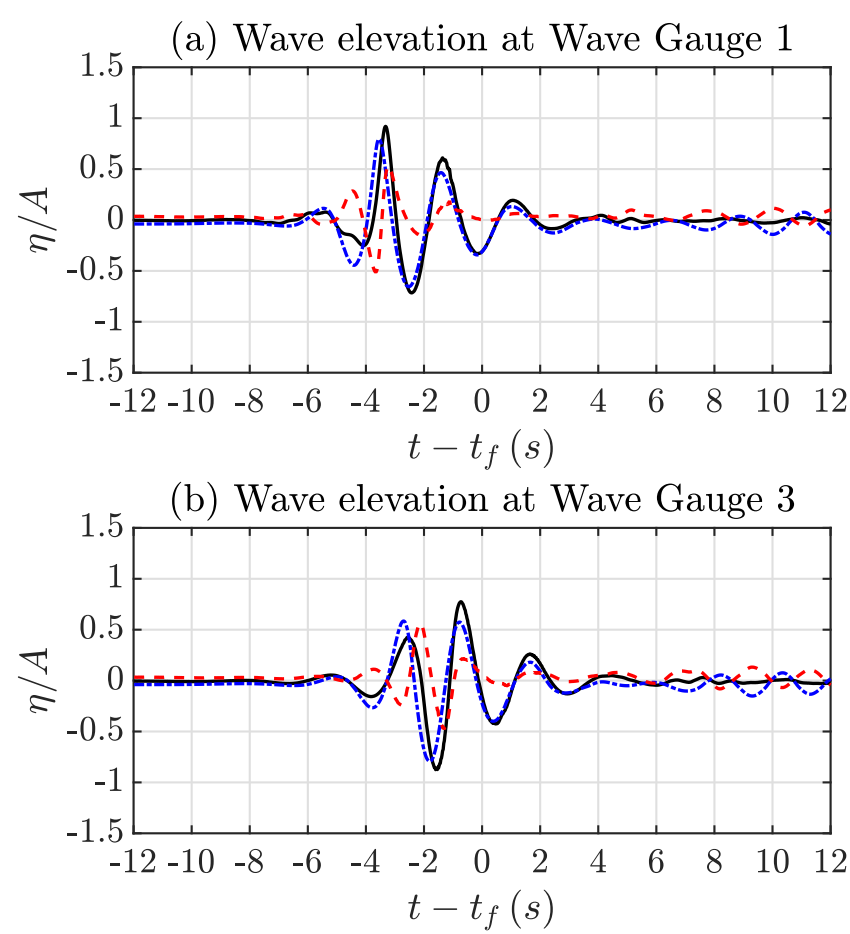

(c) Wave elevation at Wave Gauge 5

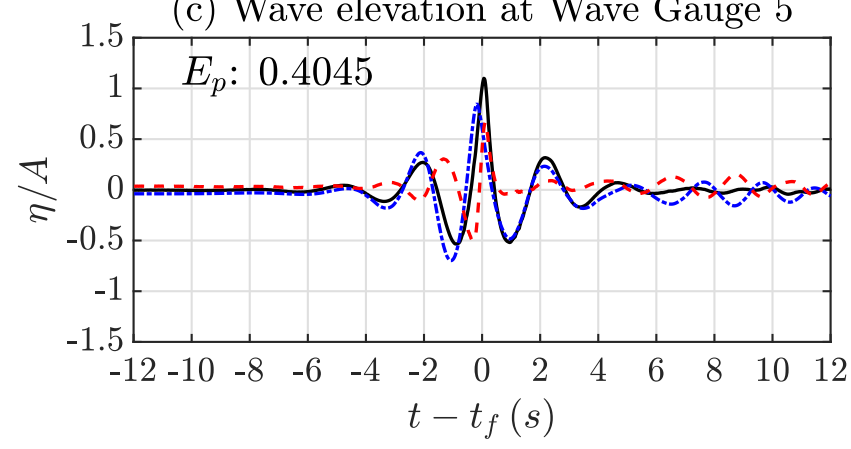

(d) Wave elevation at Wave Gauge 8

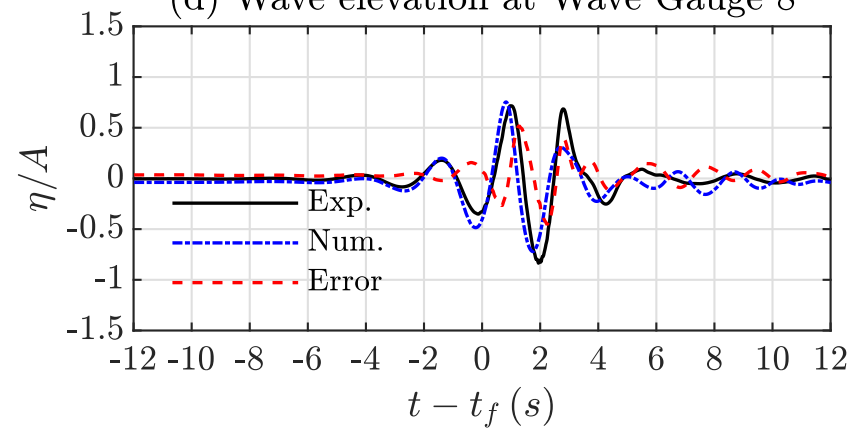

Figure 9. Comparisons between numerical results of Case 3 against experimental data at four Wave Gauges.

in order to understand the effects of wave steepness and WEC geometry on motion response and mooring force. The focused wave properties are tabulated in Table 2. Under these focused wave groups, the motions of the cylindrical moon-pool are presented in

\begin{tabular}{ccccc}
\hline ID & Case & Cell number & CPU core number & $\begin{array}{c}\text { Clock time } \\
{[\mathrm{hrs}]}\end{array}$ \\
\hline 1 & 1 & $4.5 \mathrm{M}$ & 64 & 108.5 \\
1 & 2 & $4.5 \mathrm{M}$ & 64 & 146.0 \\
1 & 3 & $4.5 \mathrm{M}$ & 64 & 142.7 \\
2 & 1 & $4.5 \mathrm{M}$ & 64 & 66.3 \\
2 & 2 & $4.5 \mathrm{M}$ & 64 & 105.3 \\
2 & 3 & $4.5 \mathrm{M}$ & 64 & 112.5 \\
\hline
\end{tabular}

Note: All cases were simulated using CPU Intel ${ }^{\circledR}$ Xeon ${ }^{\circledR}$ E5 2603 @ $1.7 \mathrm{GHz}$. The data shown in this table are the data submitted to CCP-WSI Blind Test Series 2.

Table 5. Computational time for each simulation

Fig. 10, together with the time history of the mooring forces. It clearly shows that under the condition of the same focal wave crest, the maximum amplitudes of heave, surge and pitch motions, and mooring force after focal time are similar. However, the subsequent surge motion seems to be significantly influenced by the increase of wave steepness. It can be seen from the surge time series that the floating cylinder with moon-pool is drifted away by the incident focused wave, and then gradually returns to its initial position. However, after focal time a different motion response of a hemispherical-bottom cylinder WEC in the focused waves can be found in Fig. 11, in particular for the surge and pitch motions. These difference of heave among these cases may be induced by the increasing wave steepness of focused waves.

The different motion responses of two types of WECs under the same incident waves (Case 2) in Fig. 12 is primarily due to significant differences in geometry in Figs. 1-2. It can be noted that the heave motion and mooring force of both WECs seems to be similar for both peak amplitudes, while significant differences are presented in the surge and pitch responses. For the cylinder with a moon-pool WEC, it requires longer time to return to its initial location ( $\operatorname{surge}=0 \mathrm{~m}$ ), and the pitch motion decays faster during de-focusing process when compared to that of the hemispherical bottomed WEC. This may be primarily due to the larger moment of inertia of cylindrical moon-pool WEC and viscous damping. The effect of geometry on the motion response remains unclear and needs to be further investigated. Moreover, the required computational time for each case is indicated in Table 5, along with the corresponding mesh cell and CPU core numbers. It can be noted that with the increase of wave steepness the simulation normally takes longer to complete due to the use of a fixed Courant-Friedrichs-Lewy number ( $\mathrm{CFL}=0.3$ for all cases) and the resulting smaller time step.

\subsection{Numerical wave reproduction based on experimental measurements}

In Blind Test Series 2, the signal and motion of wave paddles were not provided beforehand and the domain sizes in laboratory 

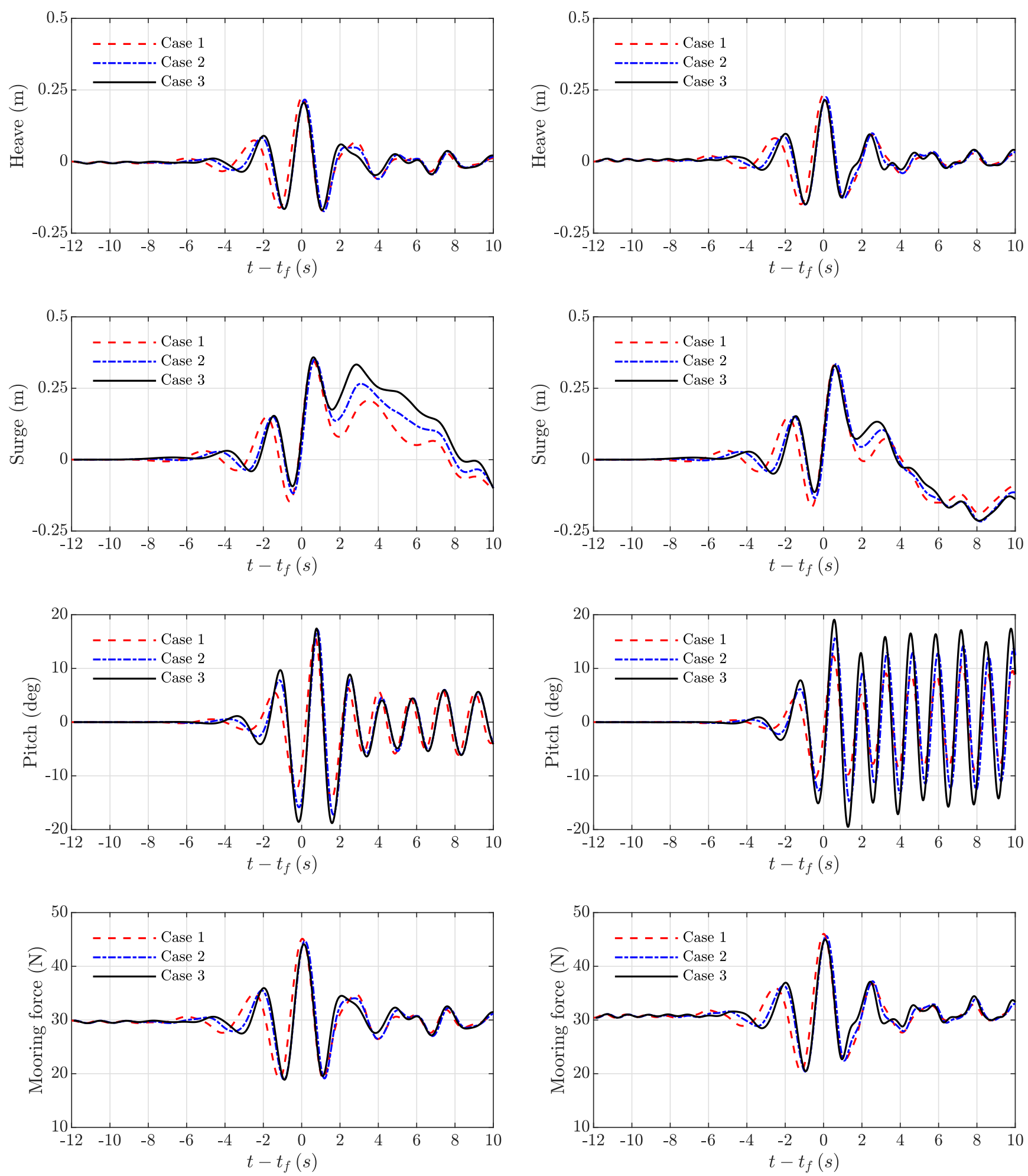

Figure 10. Heave, surge, pitch, and mooring force of the cases with a moon-pool cylinder.

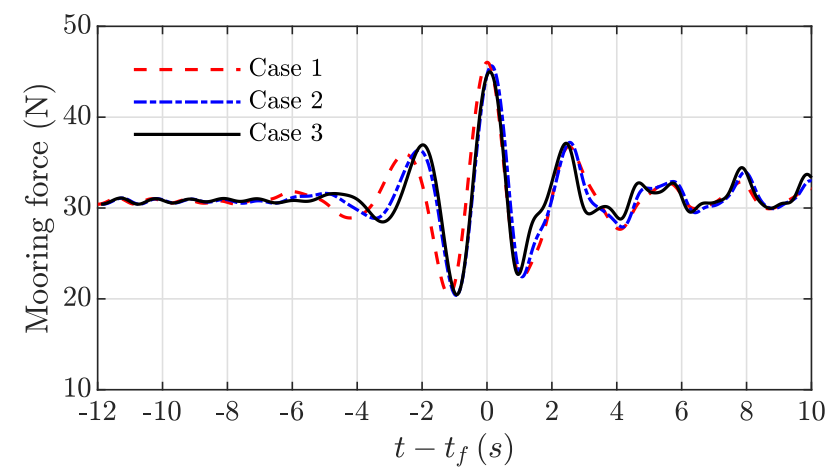

Figure 11. Heave, surge, pitch, and mooring force of the cases with a hemispherical-bottom cylinder. 

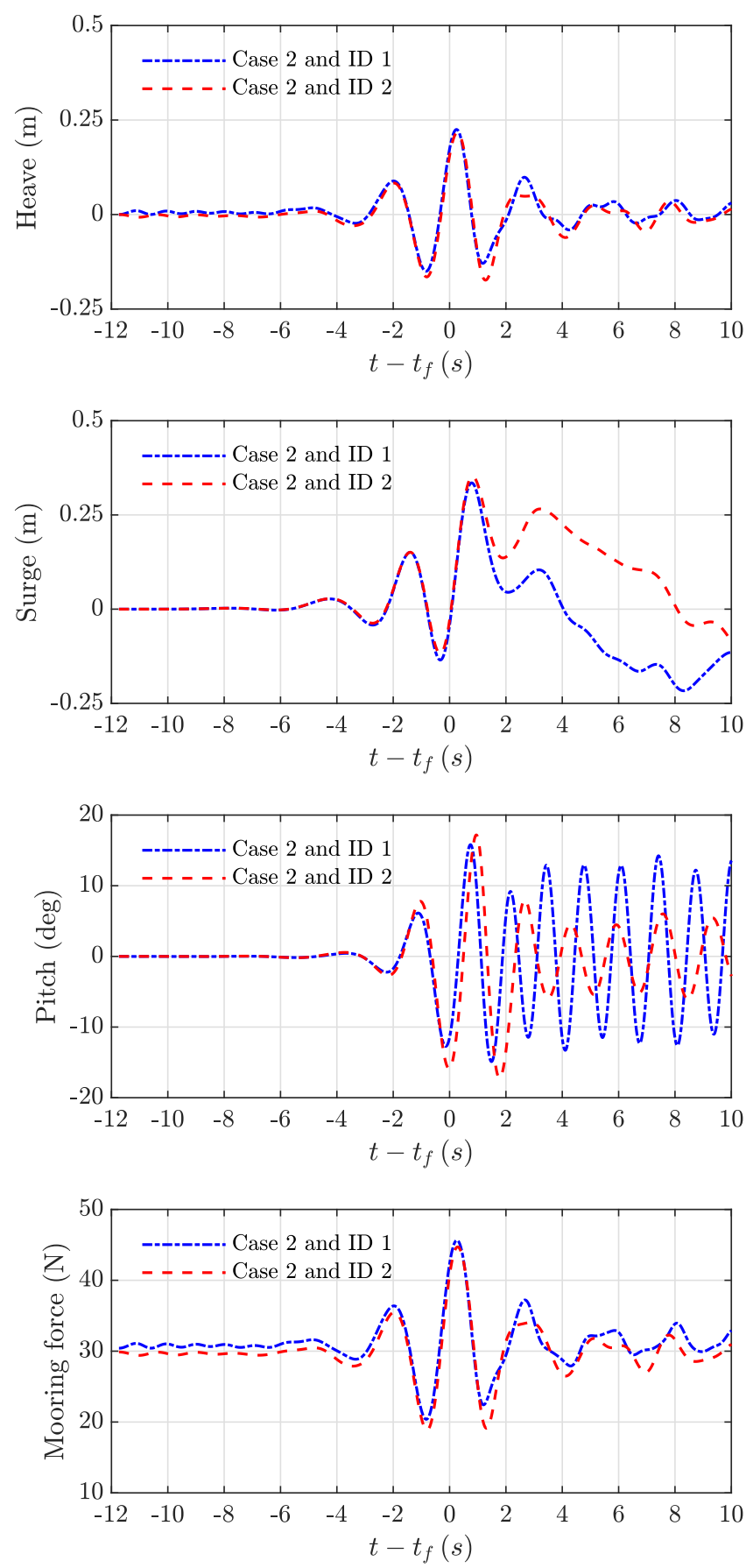

Figure 12. Comparisons of heave, surge, pitch, and mooring force of the Case 2 between hemispherical-bottom cylinder (ID 1) and cylindrical moon-pool (ID 2)

and numerical simulation are different. This leads to the difficulty in numerically reproducing the target wave profiles in laboratory on the basis of given wave parameters for the experiments, and eventually results in the discrepancy in the generated waves as shown in Figs. 7, 8, and 9. In order to accurately evaluate the survivability of WECs, an accurate reproduction of the incident waves plays a key role in determining the motion response of WECs in focused waves. Thus, aiming to reproducing the incident focused waves accurately in our numerical simulations, the focal time and locations of the target waves have been tuned to match experimental data, while other wave parameters, such as focal crest height $A$ and the wave period for the wave component at peak frequency $T_{p}$, remained the same. Here, to demonstrate their effects on the solutions, only Case 2 in Blind Test Series 2 was used as a test case.

In the numerical simulation of Case 2, the theoretical focal time and location were assumed to be at $12 \mathrm{~s}$ and $12 \mathrm{~m}$ away from the wave generation boundary, respectively. From the results presented in Fig. 8, it seems that the numerically generated focused waves have actually focused at a location downstream of the theoretical focal point and be slightly later than the theoretical focal time, as wave profile of WG5 in Fig. 8 is asymmetric and has larger wave trough around $t-t_{f}=-1 \mathrm{~s}$ compared to the experimental measurements. To correct these and to better reproduce the experiments, a trial and error tuning has been performed to determine the the actual focal time and location in the numerical wave basin and consequently the focal time and location are modified from $12 \mathrm{~s}$ and $12 \mathrm{~m}$ to $11.5 \mathrm{~s}$ and $10.5 \mathrm{~m}$, respectively. The corresponding new numerical results are demonstrated in Fig. 13, which showed better agreements with experimental measurements with smaller error (approximately from 0.2 of WG5 in Fig. 8 to 0.1 of WG5 in Fig. 13) compared with the old numerical results in Fig. 8 (Lin et al., 2019).

\subsection{Validation of motion response of WECs against released experimental WECs motions}

As the blind tests imply and has been done previously in Blind Test Series 1 (Ransley et al., 2019) and 3 (Ransley et al., 2020), the experimental results were only released after the submission of the results. Therefore the analysis on motion responses of two WECs in the previous sections have been done without comparing them with the experimental data. In this section the comparisons of original submitted numerical results and new numerical results based on the tuned wave focused point and time against experimental measurements are presented. The mooring force comparison in Figs. 15 and 16 shows that there is slight discrepancy between old pretension in mooring and experimental measurements using the suggested rest length $2.224 \mathrm{~m}$. The detailed setup of the mooring line in laboratory is demonstrated in Fig. 14, where mooring line length is around $2.199 \mathrm{~m} \pm 0.006 \mathrm{~m}$. Thus, a new rest length $2.199 \mathrm{~m}$ is used in the new numerical simulation in order to match the experimental setup. It can be seen from both Figs. 15 and 16 that the new numerical results with new rest length $2.199 \mathrm{~m}$ show better agreement with the pretension in mooring in laboratory. Other modifications of new model, such as mesh generation and parameters used in model setup, are described in Chen et al. (2020) due to the similarity of two Blind Test Series. 

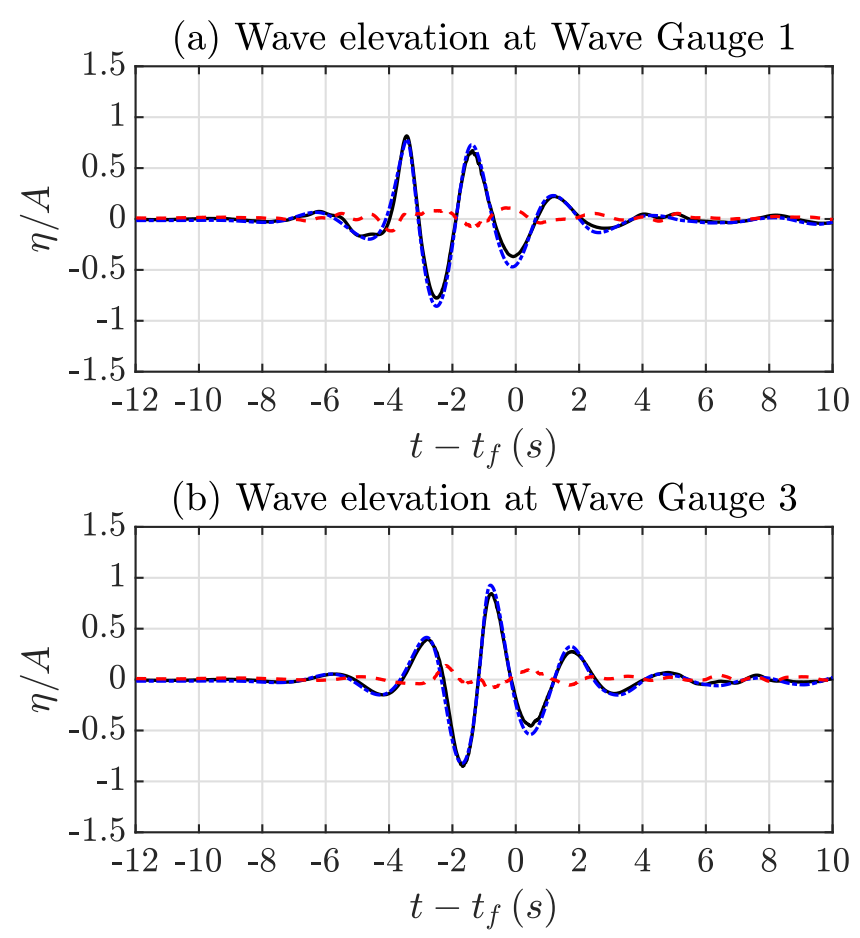

(c) Wave elevation at Wave Gauge 5

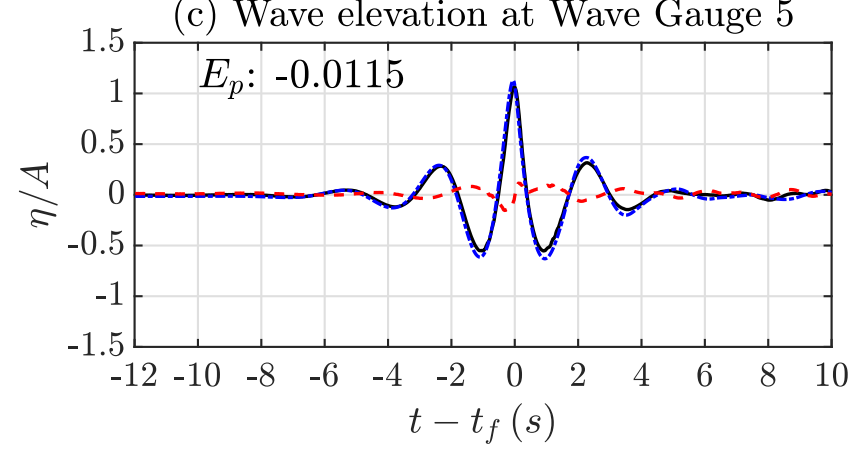

(d) Wave elevation at Wave Gauge 8

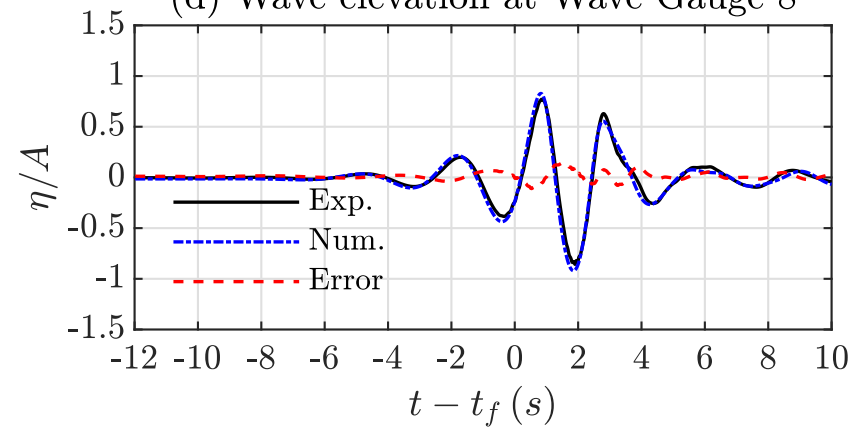

Figure 13. Comparisons between numerical results of Case 2 by tuning focal time and position against experimental data at four Wave Gauges.

With the aforementioned modifications including the new focused point and time, the new motion responses of the two WECs are shown in Figs. 15 and 16, together with the old motion response

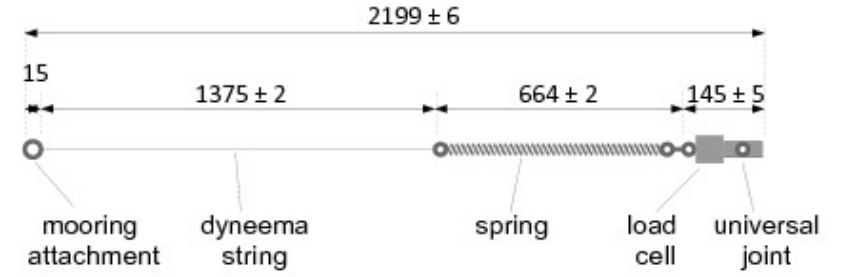

Figure 14. Details of mooring line setup in laboratory $(\mathrm{mm})$. Source: CCP-WSI Blind Test Series 2 (2020)

and released experimental measurements. It clearly demonstrates that those changes have significantly improved the accuracy of the overset model in predicting the motion response and mooring force of two WECs in focused waves, although slight discrepancies in surge and pitch motions after focal time can still be seen compared with experimental results. This may be due to larger wave trough after the focal time at WG5 in the numerical results as shown in Fig. 13. One possible reason for the differences in pitch motion periods between numerical and experimental results might be due to the fact that centre of mass has been assumed to be centre of rotation for the pitch motion in both the numerical and experimental work. However, under certain restraints such as mooring the instantaneous centre of rotation of a floating structure may not be the same as centre of mass (Costa et al., 2018).

\subsection{Discussion on 'tuning' focused waves for their reproduction in NWT}

Tuning focusing time and location for wave generation in the NWT is generally not required for simulations involving focused waves. However, in the present work, to reduce the computational time, a smaller wave tank with a different wave generation method has been used so it will be difficult to precisely reproduce the physically generated focused waves based on the theoretical focal time and location. On the other hand, as motion response of a floating structure is highly sensitive to the quality of focused waves reproduction, a trial and error process (Chen et al., 2019d) or the iterative wave generation procedure (Yan et al., 2020) are usually applied in order to achieve fair comparisons with the experimental measurements. It should be noted that the tuned focal time and location will usually differ from the theoretical values used in the wave tank tests. For future parametric studies with varying wave parameters, the wave generation methods that mimic the physical wave maker motions in Hu et al. (2016) and Chen et al. (2019a) can be applied to better reproduce the focused waves generated in wave tank experiments 

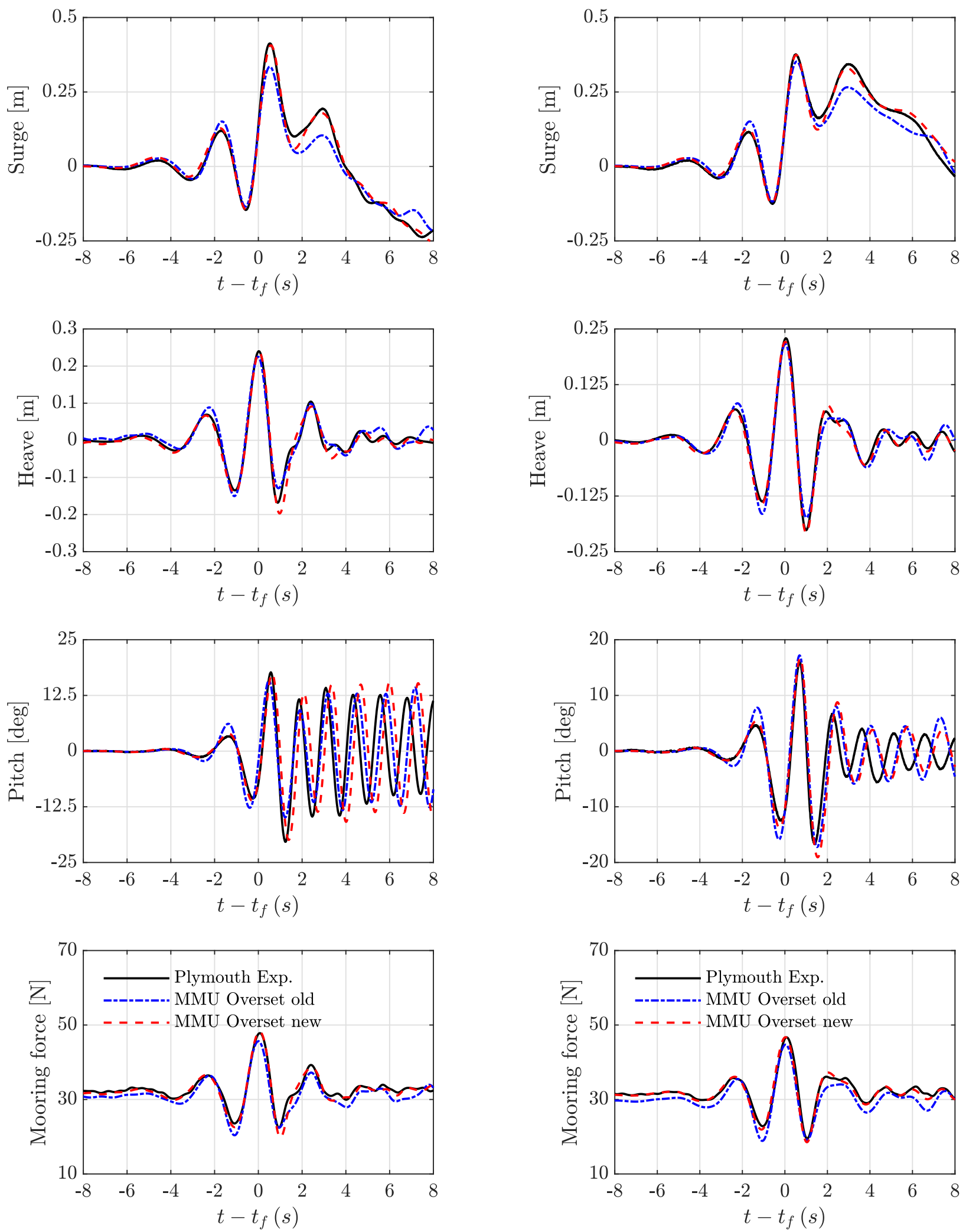

Figure 15. Comparisons of hemispherical-bottom cylinder motion response between different numerical results of Case 2 of against experimental measurements.

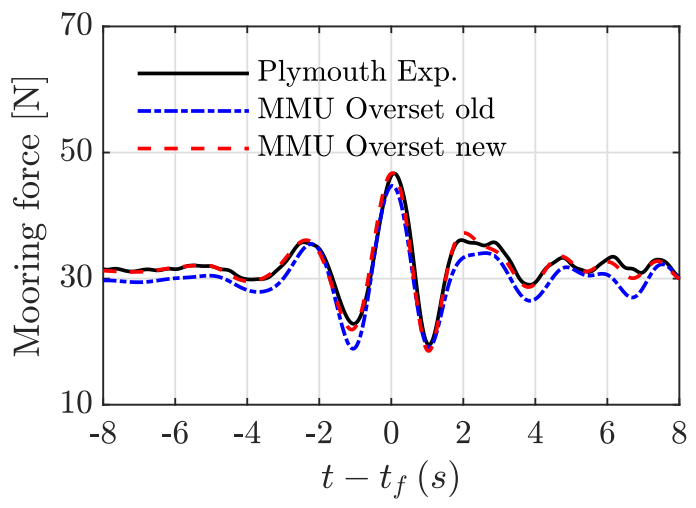

Figure 16. Comparisons of moon-pool cylinder motion response between different numerical results of Case 2 of against experimental measurements. 


\section{Conclusion}

In this paper, the numerical simulations of the two simplified point absorber WECs, namely the hemispherical-bottom cylinder and the cylinder with a moon-pool, under focused wave groups are presented, which forms a contribution to the CCP-WSI Blind Test Series 2. Overset mesh in OpenFOAM is adopted to capture the motion response of WECs in the focused wave groups with varying peak frequencies and a constant focused wave crest. It is found that the maximum amplitude and period of heave, pitch, and mooring force for the cylinder with a moon-pool are similar, while the major difference occurs at the surge motion due to the varying peak frequencies and wave steepness. The comparisons of the motions of two WECs with and without a moon-pool indicate that under the same wave groups, differences in geometry and moment of inertia may lead to distinct surge and pitch motions, although the heave and mooring force tend to be less affected. Subjected to the same wave groups, the WEC with moon-pool may drift further away (surge motion) from its initial location, while it tends to recover to its initial position faster after the focal time in terms of the pitch motion compared to those of the hemisphericalbottom WEC. The motion response of the floating WECs are highly sensitive to the accuracy of numerical wave reproduction and the instantaneous centre of rotation during wave-structure interaction under certain restraints may differ from the centre of mass, which might explain the differences in pitch motion between the numerical prediction and experimental measurements and needs to be further investigated.

\section{Acknowledgements}

This work was partially funded by the Engineering and Physical Sciences Research Council (EPSRC, UK) projects: A Zonal CFD Approach for Fully Nonlinear Simulations of Two Vessels in Launch and Recovery Operations (EP/N008839/1), Extreme Loading on FOWT under Complex Environmental Conditions (EP/T004150/1), and A CCP on Wave Structure Interaction: CCP-WSI (EP/M022382/1). The first author would also like to acknowledge the financial support from the Manchester Metropolitan University through sponsoring a Research Associate position.

\section{REFERENCES}

Baldock T, Swan C and Taylor P (1996) A laboratory study of nonlinear surface waves on water. Philosophical Transactions of the Royal Society of London. Series A: Mathematical, Physical and Engineering Sciences 354(1707): 649-676.

Berberović E, van Hinsberg NP, Jakirlić S, Roisman IV and Tropea C (2009) Drop impact onto a liquid layer of finite thickness: Dynamics of the cavity evolution. Physical Review E 79(3): 036306.

CCP-WSI Blind Test Series 2 (2020) Focused wave interactions with floating structures (CCP-WSI Blind Test Series 2).

[Online]. Available:

https://www.ccp-wsi.ac.uk/blind_test_series_2 .
Chen H, Lin Z, Qian L, Ma Z, Bai W et al. (2020) CFD simulation of wave energy converters in focused wave groups using overset mesh. International Journal of Offshore and Polar Engineering 30(01): 70-77.

Chen H, Qian L, Bai W, Ma Z, Lin Z and Xue MA (2019a) Oblique focused wave group generation and interaction with a fixed FPSO-shaped body: 3D CFD simulations and comparison with experiments. Ocean Engineering 192: 106524.

Chen H, Qian L, Ma Z, Bai W, Li Y, Causon D and Mingham C (2019b) Application of an overset mesh based numerical wave tank for modelling realistic free-surface hydrodynamic problems. Ocean Engineering 176: 97-117.

Chen H, Qian L, Ma Z, Bai W and Lin Z (2019c) CCP-WSI blind test series 3: OpenFOAM simulation of focused wave interaction with a simplified wave energy converter. In The 29th International Ocean and Polar Engineering Conference, International Society of Offshore and Polar Engineers.

Chen Q, Zang J, Ning D, Blenkinsopp C and Gao J (2019d) A 3d parallel particle-in-cell solver for extreme wave interaction with floating bodies. Ocean Engineering 179: 1-12.

Costa DdO, Fernandes AC, Sales Junior JS and Asgari P (2018) Instantaneous center of rotation in pitch response of a FPSO submitted to head waves. In ASME 2018 37th International Conference on Ocean, Offshore and Arctic Engineering, American Society of Mechanical Engineers Digital Collection.

Devolder B, Rauwoens P and Troch P (2016) Numerical simulation of a single floating point absorber wave energy converter using OpenFOAM $®$. Progress in Renewable Energies Offshore; CRC Press: Boca Raton, FL, USA : 197-205.

Devolder B, Stratigaki V, Troch P and Rauwoens P (2018) CFD simulations of floating point absorber wave energy converter arrays subjected to regular waves. Energies 11(3): 641.

Domínguez JM, Crespo AJ, Hall M, Altomare C, Wu M, Stratigaki V, Troch P, Cappietti L and Gómez-Gesteira M (2019) SPH simulation of floating structures with moorings. Coastal Engineering 153: 103560.

Hann M, Greaves D and Raby A (2015) Snatch loading of a single taut moored floating wave energy converter due to focussed wave groups. Ocean Engineering 96: 258-271.

Higuera P, Lara JL and Losada IJ (2013a) Realistic wave generation and active wave absorption for navier-stokes models: Application to OpenFOAM $®$. Coastal Engineering 71: 102-118.

Higuera P, Lara JL and Losada IJ (2013b) Simulating coastal engineering processes with OpenFOAM $₫$. Coastal Engineering 71: 119-134.

Hu ZZ, Causon D, Mingham C and Qian L (2011) Numerical simulation of floating bodies in extreme free surface waves. Natural Hazards and Earth System Sciences 11(2): 519-527.

Hu ZZ, Greaves D and Raby A (2016) Numerical wave tank study of extreme waves and wave-structure interaction using OpenFOAM $₫$. Ocean Engineering 126: 329-342. 
Lin Z, Qian L, Ma Z, Chen H, Causon D and Mingham C (2019) Simulation of focused wave impact on point absorber wave energy converters - CCP-WSI Blind Test Series 2. In 13th European Wave and Tidal Energy Conference EWTEC 2019.

Ma Z, Qian L, Martinez-Ferrer P, Causon D, Mingham C and Bai W (2018) An overset mesh based multiphase flow solver for water entry problems. Computers \& Fluids 172: 689-705.

Palm J, Eskilsson C, Paredes GM and Bergdahl L (2016) Coupled mooring analysis for floating wave energy converters using CFD: Formulation and validation. International Journal of Marine Energy 16: 83-99.

Ransley E, Brown S, Hann M, Greaves D, Windt C, Ringwood J, Davidson J, Schmitt P, Yan S, Wang JX, Wang JH, Ma Q, Xie Z, Giorgi G, Hughes J, Williams A, Masters I, Lin Z, Chen H, Qian L, Ma Z, Chen Q, Ding H, Zang J, van Rij J, Yu Y, Li Z, Bouscasse B, Ducrozet $G$ and Bingham $H$ (In review) Focused wave interactions with floating structures: A blind comparative study. Proceedings of the Institution of Civil Engineers Engineering and Computational Mechanics .

Ransley E, Greaves D, Raby A, Simmonds D and Hann M (2017) Survivability of wave energy converters using CFD. Renewable Energy 109: 235-247.

Ransley E, Yan S, Brown S, Hann M, Graham D, Windt C, Schmitt P, Davidson J, Ringwood J, Musiedlak PH, Wang J, Wang J, Ma Q, Xie Z, Zhang N, Zheng X, Giorgi G, Chen H, Lin Z, Qian L, Ma Z, Bai W, Chen Q, Zang J, Ding H, Cheng L, Zheng J, Gu H, Gong X, Liu Z, Zhuang Y, Wan D, Bingham H and Greaves D (2020) A blind comparative study of focused wave interactions with floating structures (CCP-WSI Blind Test Series 3). International Journal of Offshore and Polar Engineering 30: $1-10$.

Ransley E, Yan S, Brown S, Mai D T Graham, Ma Q, Musiedlak PH, Engsig-Karup A, Eskilsson C, Li Q, Wang J, Xie Z, Sriram V, Stoesser T, Zhuang Y, Li Q, Wan D, Chen G, Chen H, Qian L, Ma Z, Mingham C, Causon D, Gatin I, Jasak H, Vukčević V, Downie S, Higuera P, Buldakov E, Stagonas D, Chen Q, Zang J and Greaves D (2019) A blind comparative study of focused wave interactions with a fixed FPSO-like structure (CCP-WSI Blind Test Series 1). International Journal of Offshore and Polar Engineering 29(2): 113-127.

Tromans PS, Anaturk AR, Hagemeijer P et al. (1991) A new model for the kinematics of large ocean waves-application as a design wave. In The First International Offshore and Polar Engineering Conference, International Society of Offshore and Polar Engineers.

Westphalen J, Greaves D, Hunt-Raby A, Williams CJ, Taylor PH, Hu Z, Causon D, Mingham C, Stansby P, Rogers B et al. (2010) Numerical simulation of wave energy converters using eulerian and lagrangian cfd methods. In The Twentieth International Offshore and Polar Engineering Conference, International Society of Offshore and Polar Engineers.
Yan S, Wang J, Wang J, Ma Q, Xie Z et al. (2020) CCP-WSI blind test using qaleFOAM with an improved passive wave absorber. International Journal of Offshore and Polar Engineering 30(01): 43-52.

Yu YH and Li Y (2013) Reynolds-averaged navier-stokes simulation of the heave performance of a two-body floating-point absorber wave energy system. Computers \& Fluids 73: 104-114. 